Classification

Physics Abstracts

87.30G $-64.60 \mathrm{C}-75.10 \mathrm{H}$

\title{
Storage capacity of memory networks with binary couplings
}

\author{
Werner Krauth ( $\left.{ }^{1}\right)$ and Marc Mézard ( $\left.{ }^{2}\right)$ \\ (1) Laboratoire de Physique Statistique de l'Ecole Normale Supérieure (*), 24 rue Lhomond, \\ 75231 Paris Cedex 05, France \\ (2) Laboratoire de Physique Théorique de l'Ecole Normale Supérieure, 24 rue Lhomond, 75231 \\ Paris Cedex 05, France
}

(Reçu le 17 mai 1989, accepté le 7 juillet 1989)

\begin{abstract}
Résumé. - Nous étudions le nombre $p$ de prototypes aléatoires non biaisés qui peuvent être mémorisés dans un réseau de $N$ neurones utilisé comme mémoire associative, dans le cas où les efficacités synaptiques ne peuvent prendre que les valeurs \pm 1 . Nous trouvons une solution avec une étape de brisure de symétrie des répliques à la Parisi. Cette solution prédit une capacité optimale $\alpha_{\mathrm{c}}=p / N \approx 0,83$ qui semble en bon accord avec les résultats numériques connus.
\end{abstract}

\begin{abstract}
We study the number $p$ of unbiased random patterns which can be stored in a neural network of $N$ neurons used as an associative memory, in the case where the synaptic efficacies are constrained to take the values \pm 1 . We find a solution with one step of replica symmetry breaking à la Parisi. This solution gives a critical capacity $\alpha_{\mathrm{c}}=p / N \sim 0.83$ which seems to agree with known numerical results.
\end{abstract}

\section{Introduction.}

Consider a network of $N$ binary neurons $\sigma_{i}= \pm 1$, coupled through synapses $J_{i j}$, and evolving in time according to the zero temperature Monte Carlo dynamics (sequential or parallel) :

$$
\sigma_{i}^{t+1}=\operatorname{Sign}\left(\sum_{j} J_{i j} \sigma_{j}^{t}\right) .
$$

The network is used as an associative memory to store $p$ patterns which are $N$ bit words, $\xi_{i}^{\mu}= \pm 1, i=1, \ldots, N ; \mu=1, \ldots, p$. An important question which immediately arises is what is the capacity of such a network? This is usually quantified by the number of random patterns which can be memorised in the limit $N \rightarrow \infty$. A lot of work has already been devoted to this capacity problem, firstly in the case of some specific learning rules. For instance with Hebb's rule $J_{i j}=(1 / N) \Sigma_{\mu} \xi_{i}^{\mu} \xi_{j}^{\mu}$ the asymptotic capacity is $\alpha_{\mathrm{c}}=p / N \sim 0.14[1,2]$.

(*) Laboratoire associé au CNRS et à l’Université Pierre et Marie Curie (URA 1306). 
A major breakthrough in this problem was achieved by Gardner who showed how to calculate the optimal capacity $\alpha$, that is the number of patterns per neuron which can be stored by the best learning rule [3]. Gardner's calculation imposed no constraint on the couplings $J_{i j}$ and gave an optimal capacity for unbiased random patterns of $\alpha_{\mathrm{c}}=2$. This result confirms an old analysis of Cover [4] and allows to extend it to more complicated distributions of patterns with bias or correlations [3, 5].

Another direction in which a similar result is clearly needed is the case where the synapses $J_{i j}$ are constrained. The extreme case where $J_{i j}$ can take only two volumes $J_{i j}= \pm 1$ is of particular interest for at least two reasons :

- first of all one can count the number of bits used in the synapses, $N^{2}$, and compare it to the number of stored bits which is $\alpha N^{2}$ for unbiased random patterns;

- secondly, it is clear that for practical applications one does not want to require an infinite precision for the $J_{i j}$ 's but one wants to code them on a small number of bits. The present case is just the extreme one of one bit per synapse $\left(^{1}\right)$. For the specific case of the clipped Hebb's rule $J_{i j}=\operatorname{Sign}\left(\Sigma_{\mu} \xi_{i}^{\mu} \xi_{j}^{\mu}\right)$ Sompolinsky [6] found a capacity of $\alpha \sim 0.1$.

In this paper we consider the problem of the learning capacity for unbiased random patterns, with $J_{i j}$ constrained to the values \pm 1 , independently of any specific choice for the learning rule. This problem has already been considered by Gardner and Derrida [5] who found a replica symmetric solution giving $\alpha_{\mathrm{c}}=4 / \pi$ but showed that replica symmetry must be broken. The solution we give here uses one step of replica symmetry breaking in Parisi's hierarchical scheme and predicts $\alpha_{\mathrm{c}}=0.83$. Thus the effects of replica symmetry breaking are drastic. This is due to a first order phase transition.

\section{Replica formulation.}

The starting point of the computation follows previous approaches [5]. In a $N+1$ neuron system we consider the $N$ couplings $J_{0 i}=J_{i}, i=1, \ldots, N$, incoming onto a given neuron $i=0$. For a pattern $\mu$ to be learnt one needs that it be a fixed point of the dynamics :

$$
\xi_{0}^{\mu}=\operatorname{Sign}\left(\sum_{k=1}^{N} J_{k} \xi_{k}^{\mu}\right)
$$

which we write as $\Sigma_{k} J_{k} \eta_{k}^{\mu}>0$, where $\eta_{k}^{\mu}=\xi_{0}^{\mu} \xi_{k}^{\mu}$ are independent random variables, taking values \pm 1 with probability $1 / 2$. It is useful to generalize this condition to $\Sigma_{k} J_{k} \eta_{k}^{\mu}>\kappa \sqrt{N}$ where $\kappa$ is a stability parameter. When $\kappa$ is positive any configuration which differs from $\xi^{\mu}$ in less than $\kappa \sqrt{N} / 2$ bits will flow towards it. In fact it has been shown that the radius of the attraction basin is much larger (of order $N$ ) and increases with $\kappa[7,8]$.

For each set of couplings we define the energy as the number of patterns which are not memorized :

$$
E[J, \eta]=\sum_{\mu=1}^{p} \theta\left(\kappa-\frac{1}{\sqrt{N}} \sum_{i=1}^{N} J_{i} \eta_{i}^{\mu}\right) .
$$

Following Gardner and Derrida [5] we introduce, for a given set of patterns $\eta_{k}^{\mu}$, the partition function at temperature $T=1 / \beta$ :

$$
Z=\sum_{\left\{J_{i}\right\}} \mathrm{e}^{-\beta E[J, \eta]}
$$

(1) Intermediate cases have been studied by Gutfreund et al. (private communication). 
The physical properties of the problem defined by this partition function nicely map onto the desired properties of the learning problem in the zero temperature limit :

- the internal energy at zero temperature is the number of ill-memorized patterns (those with $\Sigma_{k} J_{k} \eta_{k}^{\mu}<\kappa \sqrt{N}$ ) for the optimal configuration of $J_{i}$ 's ;

- the patterns can be learnt if and only if the zero temperature internal energy vanishes ; in this case the zero temperature entropy is the logarithm of the number of coupling configurations which can learn all the patterns with a stability larger than $\kappa$.

As we shall see, although we are mostly interested in the zero temperature limit, it is extremely useful to consider the problem also at finite temperatures.

As we want to compute extensive thermodynamics quantities, we expect that these will be self-averaging, so that the properties of one typical sample will be obtained in the thermodynamic limit by the quenched average of $\log Z$. This is computed with the replica method [9] which gives in the present case [5] (denoting by a bar the average over the distribution of patterns) :

$$
\bar{Z}^{n}=\prod_{1 \leqslant a<b \leqslant n}\left(\int_{-i \infty}^{i \infty} \frac{\mathrm{d} \hat{Q}_{a b}}{2 \pi / N} \int_{-\infty}^{+\infty} \mathrm{d} Q_{a b}\right) \mathrm{e}^{N\left[-\sum_{a<b} Q_{a b} Q_{a b}+G_{1}(Q)+\alpha G_{0}(Q)\right]}
$$

where

$$
\begin{array}{r}
G_{1}(\hat{Q})=\log \left(\sum_{\left\{J^{a}= \pm 1\right\}} \mathrm{e}^{\sum_{a<b} Q_{a b} J^{a} J^{b}}\right) \\
G_{0}(Q)=\log \left(\prod_{a=1}^{n}\left[\int_{-i \infty}^{i \infty} \frac{\mathrm{d} \lambda^{a}}{2 \pi}\left(\mathrm{e}^{-\beta} \int_{-\infty}^{+\infty} \mathrm{d} t^{a}+\left(1-\mathrm{e}^{-\beta}\right) \int_{\kappa}^{\infty} \mathrm{d} t^{a}\right)\right]+\right. \\
\left.+\mathrm{e}^{\sum_{a=1}^{n}\left(\lambda^{a} t^{a}+\frac{1}{2}\left(\lambda^{a}\right)^{2}+\frac{1}{2} \sum_{a \neq b} Q^{a b} \lambda^{a} \lambda^{b}\right)}\right)
\end{array}
$$

$Q_{a b}$ is the natural order parameter which measures the overlap of the configurations of couplings in two replicas : $Q_{a b}=(1 / N) \Sigma_{i} J_{i}^{a} J_{i}^{b}$. The interpretation of $\hat{Q}_{a b}$ involves the probability of one constraint to be strict $\Sigma_{k} J_{k} \eta_{k}^{\mu}=\kappa \sqrt{N}$ and is more complicated [10].

Starting from the representation $(5,6)$, one can study various types of approximations or Ansätze for the form of the order parameters $Q_{a b}$ and $\hat{Q}_{a b}$ on the saddle point of (5). Hereafter we shall consider successively the annealed approximation, the replica symmetric solution, and the replica symmetry breaking (r.s.b.) solution.

\section{Annealed approximation.}

This is obtained by taking $n=1$ and approximating the quenched free energy density $F / N=-\{T / N\} \overline{\log Z}$ by the annealed average :

$$
\frac{\mathrm{F}_{\mathrm{ann}}}{N}=-\frac{T}{N} \log (\bar{Z})=-T\left[\log 2+\alpha \log \left(\mathrm{e}^{-\beta}+\left(1-\mathrm{e}^{-\beta}\right) H(\kappa)\right)\right]
$$

where $H(\kappa)$ is an error function defined as :

$$
H(\kappa)=\int_{\kappa}^{\infty} \frac{\mathrm{d} t}{\sqrt{2 \pi}} \mathrm{e}^{-t^{2} / 2}
$$


From (7) we see that the density of internal energy vanishes in the zero temperature limit, while the density of entropy at zero temperature is :

$$
\frac{S_{\mathrm{ann}}(T=0)}{N}=\log 2+\alpha \log H(\kappa) .
$$

As is well known, because of the concavity the logarithm, the annealed free energy is a lower bound to the correct quenched free energy. From this and the fact that the quenched free energy cannot be negative we obtain a lower bound on the zero temperature energy density [11]. This implies in particular, using (9), that :

$$
\alpha_{\mathrm{c}} \leqslant \frac{\log 2}{-\log H(\kappa)} \equiv \alpha_{\mathrm{A}}(\kappa) \text {. }
$$

For stability $\kappa=0$, this gives $\alpha_{\mathrm{c}} \leqslant 1$ which corresponds to the bound from the theory of information.

\section{Replica symmetric solution.}

In order to compute the correct quenched free energy in (5), (6) we want to take the $n \rightarrow 0$ limit. To make this step we need an Ansatz on the form of $Q_{a b}$ and $\hat{Q}_{a b}$ on the saddle point. The simplest approximation is the replica symmetric one where $Q_{a b}=q$ and $\hat{Q}_{a b}=\hat{q}$. This has been studied by Gardner and Derrida [5] and gives

$$
\begin{aligned}
& G_{\mathrm{r} . \mathrm{s} .}(q, \hat{q}, \beta) \equiv \frac{1}{N n} \log \bar{Z}^{n}=\frac{1}{2} q \hat{q}-\frac{\hat{q}}{2}+ \\
& \quad+\int \mathrm{D} z \log (2 \cosh z \sqrt{\hat{q}})+\alpha \int \mathrm{D} z \log \left[\mathrm{e}^{-\beta}+\left(1-\mathrm{e}^{-\beta}\right) H\left(\frac{\kappa+z \sqrt{q}}{\sqrt{1-q}}\right)\right]
\end{aligned}
$$

where $\mathrm{D} z$ is a Gaussian integration measure :

$$
\mathrm{D} z \equiv \frac{\mathrm{d} z}{\sqrt{2 \pi}} \mathrm{e}^{-z^{2} / 2}
$$

and $q$, and $\hat{q}$ are obtained by solving the saddle point equations $\partial G_{0} / \partial q=\partial G_{0} / \partial \hat{q}=0$.

Solving these equations one finds the following typical properties. The zero temperature energy is zero for $\alpha \leqslant \alpha_{E}(\kappa)$, and becomes strictly positive above. $\alpha_{E}(\kappa)$ is the point where the value of the overlap $q$ goes to one at zero temperature. From this argument one would get a capacity $\alpha_{E}(0)=4 / \pi$. However there exists a second characteristic line $\alpha_{S}(\kappa)<\alpha_{E}(\kappa)$ such that, for $\alpha$ larger than $\alpha_{S}(\kappa)$, the zero temperature entropy becomes negative. Firstly this implies that the replica symmetric approximation is wrong at least for $\alpha>\alpha_{S}(\kappa)$, since the entropy cannot become negative in such a problem with a finite number $\left(2^{N}\right)$ of possible configurations. Secondly this gives a second possible value of the capacity, namely $\alpha_{S}(0) \cong 0.83$, since the vanishing of the zero temperature entropy just means that there are no longer any accessible configurations of the couplings for $T$ going to zero.

As the replica symmetric approximation gives an incorrect solution, it is natural to study its stability against replica symmetry breaking, i.e. to look whether the replica symmetric saddle point is locally stable. This calculation has been described in detail by Gardner and Derrida [5, appendix 2] for the spherical model and carries over in a straightforward manner to the Ising-type model considered here. For this latter model it was already noted by Gardner and Derrida [5] that for $\kappa=0$ the replica symmetric solution becomes unstable for some value 
$\alpha_{\text {AT }}$ with $0<\alpha_{\text {AT }}<4 / \pi$. We have determined numerically this value for zero temperature. We find that the replica symmetric solution becomes unstable at $\alpha_{\mathrm{AT}}(\kappa=0) \cong 1.015$.

\section{Physical discussion.}

Concerning the capacity problem, given by the behaviour at $T=0$, the picture which we get from the previous two sections is somewhat confusing. There are four special values of $\alpha$. Keeping for simplicity to the case $\kappa=0$, we encounter starting from small $\alpha$ the following special points :

$-\alpha_{\mathrm{S}} \cong 0.83$ : for $\alpha>\alpha_{\mathrm{S}}$ the zero temperature entropy of the replica symmetric saddle point is negative. This is one candidate value for the critical capacity. Beyond this point, the replica symmetric solution, although locally stable, is surely wrong ;

$-\alpha_{\mathrm{A}}=1$ : upper bound of the critical capacity obtained from the annealed approximation ;

$-\alpha_{\mathrm{AT}} \cong 1.015$ : for $\alpha>\alpha_{\mathrm{AT}}$ the replica symmetric solution becomes locally unstable ;

$-\alpha_{\mathrm{E}}=4 / \pi \cong 1.27$ : for $\alpha>\alpha_{\mathrm{E}}$ the zero temperature energy becomes strictly positive and $q \rightarrow 1$. This is a second candidate value for the critical capacity.

We shall see below from a physical argument that the transition to a non-zero ground state energy must take place at a value of $q$ which is much lower than $q=1$. This calls for a first order transition to a r.s.b. solution with two parameters $q_{0}$ and $q_{1}$ instead of $q$, where $q_{0}$ is close to the replica symmetric value, and $q_{1}$ is close to one.

In order to understand this effect, one can resort to some other kind of approximation scheme : if we relax the constraint $J_{i}= \pm 1$ and keep the $J_{i}$ free with the only global constraint $\Sigma_{i} J_{i}^{2}=N$, we go to a spherical model. This is the model which has been solved exactly by Gardner [3]. The replica symmetric solution is exact and gives a critical capacity $\alpha_{c}(\kappa)$, with $\alpha_{c}(0)=2$, which has been confirmed numerically [12]. The basic idea of Gardner's calculation is as follows : on the surface of the sphere $\mathbf{J}^{2}=\Sigma_{i} J_{i}^{2}=N$, the vectors $\mathbf{J}$ with zero energy (defined in (3)) constitute a subspace $f$, the surface of which is a fraction $f$ of the total surface of the sphere. The typical value of $f$ is then computed with the replica method. In our case of binary couplings the correct sets of couplings are the points which belong both to $\mathrm{f}$ and to the hypercube $J_{i}= \pm 1$. As this hypercube has $2^{N}$ points, we can expect that the critical $\alpha$ will be obtained when f contains typically one point of the hypercube, which should occur around $f \sim 1 / 2^{N}$. From Gardner's paper this happens (for $\kappa=0$ ) at $\alpha \cong 0.85$; furthermore it is most interesting to notice that for this value of $\alpha$, the typical overlap of two spherical $\{J\}$ configurations in $\mathrm{f}$ is $q \sim 0.5$.

It is reasonable to expect that the above argument gives a good approximation to the critical capacity, because the correlation of $f$ with the hypercube can be neglected. In fact, as explained in reference [18], the critical capacity of the present model is the same for any (continuous or discrete) distribution of patterns with $\bar{\xi}_{i}^{\mu}=0$ and $\overline{\left(\xi_{i}^{\mu}\right)^{2}=1}\left({ }^{2}\right)$. In the case of a continuous distribution the patterns $\xi^{\mu}$ are evidently no longer confined to the hypercube ; the hyperplanes defining $f$ are not correlated with the hypercube either. The only reason which prevents the prediction $\alpha_{c} \cong 0.85$ to be strictly exact is therefore the fact that the shape of $\mathrm{f}$ itself is not totally random (for $\alpha<1$, there are no constraints in the subspace orthogonal

(2) The special case where the patterns $\xi^{\mu}$ are normalized Gaussian variables has thus the same solution (in the $N \rightarrow \infty$ limit) as the one where the patterns are binary $\xi^{\mu}= \pm 1$. This has been used to diminish the finite size effects in numerical simulations of this problem with small values of $N$ [18]. 
to all the patterns). A somewhat similar approximation has been attempted in the case of the SK model [13].

The main result we get from this analysis is that when $f$ becomes of order $2^{-N}$ the typical overlap of two configurations is $q \sim 0.5$, therefore much lower than 1 . This same effect is found in the replica symmetric approximation: when $\alpha=\alpha_{S}(0)$ (vanishing of the zero temperature entropy), we get a replica symmetric $q \cong 0.56$, which is not too far from the above value of 0.5 .

\section{Replica symmetry breaking.}

We have looked for solutions of (5), (6) with one stage of replica symmetry breaking in the hierarchical scheme of Parisi [14]. In such a solution ergodicity is broken, the Gibbs state is decomposed into pure states $\alpha$ of relative weights $P_{\alpha}$, the Gibbs expectation value of an observable $O$ being :

$$
\langle O\rangle=\sum_{\alpha} P_{\alpha}\langle O\rangle_{\alpha}
$$

The breaking à la Parisi involves five order parameters $q_{0}, q_{1}, \hat{q}_{0}, \hat{q}_{1}, m$; the physical interpretation of $q_{0}, q_{1}, m$, is :

$$
\begin{aligned}
q_{0} & =\frac{1}{N} \overline{\sum_{i}\left\langle J_{i}\right\rangle_{\alpha}\left\langle J_{i}\right\rangle_{\beta}} \quad \alpha \neq \beta \\
q_{1} & =\frac{1}{N} \sum_{i} \overline{\left\langle J_{i}\right\rangle_{\alpha}^{2}} \\
m & =1-\sum_{\alpha} \bar{P}_{\alpha}^{2} .
\end{aligned}
$$

In terms of these order parameters we get the solution at $1^{\text {st }}$ stage of replica symmetry breaking $G_{\text {rsb }}^{(1)}$

$$
\begin{aligned}
& G_{\mathrm{rsb}}^{(1)}\left(q_{0}, \hat{q}_{0}, q_{1}, \hat{q}_{1}, m\right) \equiv \frac{1}{N n} \log \bar{Z}^{n}= \\
& \begin{aligned}
\frac{1}{2}\left[m q_{0} \hat{q}_{0}+(1-m) q_{1} \hat{q}_{1}-\hat{q}_{1}\right] \\
+\frac{1}{m} \int \mathrm{D} z_{0} \log \left(\int \mathrm{D} z_{1}\left[2 \cosh \left(z_{0} \sqrt{\hat{q}_{0}}+z_{1} \sqrt{\hat{q}_{1}-\hat{q}_{0}}\right)\right]^{m}\right) \\
+\frac{\alpha}{m} \int \mathrm{D} z_{0} \log \left(\int \mathrm { D } z _ { 1 } \left[\mathrm{e}^{-\beta}+\left(1-\mathrm{e}^{-\beta}\right)\right.\right. \\
\left.\times H\left(\frac{\kappa+z_{0} \sqrt{q_{0}}+z_{1} \sqrt{q_{1}-q_{0}}}{\sqrt{1-q_{1}}}\right)\right] m
\end{aligned}
\end{aligned}
$$

The five parameters $q_{0}, q_{1}, \hat{q}_{0}, \hat{q}_{1}, m$, must be computed through the saddle points equations :

$$
0=\frac{\partial G_{\mathrm{rsb}}^{(1)}}{\partial q_{0}}=\frac{\partial G_{\mathrm{rsb}}^{(1)}}{\partial q_{1}}=\frac{\partial G_{\mathrm{rsb}}^{(1)}}{\partial \hat{q}_{0}}=\frac{\partial G_{\mathrm{rsb}}^{(1)}}{\partial \hat{q}_{1}}=\frac{\partial G_{\mathrm{rsb}}^{(1)}}{\partial m}
$$


Stationarity with respect to $m$ is not necessarily required if one looks eventually for a continuous order parameter function $q(x)$ [19], but it is useful at first step, and it is absolutely necessary in the case where there is a first order transition as will happen here.

We have tried to solve these equations numerically. For $q_{1}<1$, we have not found a solution to all the five equations, except at $q_{0}=q_{1}$, or $m=0$ (replica symmetric solution). For fixed $m$ (that is, forgetting the last equation in (16)) there can be one or two r.s.b. solutions. If $\alpha>\alpha_{\mathrm{AT}}(\kappa)$, there are two solutions, one of which $\left(q_{0}^{(1)}, q_{1}^{(1)}\right)$ bifurcates continuously from the replica symmetric one at $\alpha=\alpha_{\mathrm{AT}}(\kappa)$, while the second one $\left(q_{0}^{(2)}, q_{1}^{(2)}\right)$ satisfies $q_{0}^{(2)}<q_{0}^{(1)}<q_{1}^{(1)}<q_{1}^{(2)}$. This second solution still exists below $\alpha_{\mathrm{AT}}(\kappa)$. Adding the last equation $\partial G_{\mathrm{rsb}}^{(1)} / \partial m=0$ in order to find the optimal $m$, we find no solution except in a region where $q_{1} \rightarrow 1$ at finite $T$.

We have therefore studied directly, what happens for $q_{1}=1$ (at finite temperature). The saddle point equation then implies that $\hat{q}_{1}=\infty$. It turns out that in this limit the expression for $G_{\mathrm{rsb}}^{(1)}$ simplies to :

$$
G_{\mathrm{rsb}}^{(1)}\left(q_{0}, \hat{q}_{0}, 1, \infty, m, \beta\right)=\frac{1}{m} G_{\mathrm{r} . \mathrm{s.}}\left(q_{0}, m^{2} \hat{q}_{0}, \beta m\right)
$$

where $G_{\text {r.s. }}$ is the replica symmetric expression (11) of $1 /(N n) \log Z^{n}$. We can easily solve the saddle point equations : stationarity with respect to $q_{0}$ and $\hat{q}_{0}$ implies that $q_{0}=q$ and $\hat{q}_{0}=\hat{q} / m^{2}$, where $q$ and $\hat{q}$ are the replica symmetric order parameters at inverse temperature $\beta m$.

Stationarity with respect to $m$ gives :

$$
S_{\text {r.s. }}\left(q_{0}, m^{2} \hat{q}_{0}, \beta m\right)=S_{\text {r.s. }}(q, \hat{q}, \beta m)=0
$$

which means that $\beta m$ must be equal to $1 / T_{\mathrm{c}}$, where $T_{\mathrm{c}}$ is the temperature where the replica symmetric entropy vanishes. Therefore we get :

- if $\alpha<\alpha_{\mathrm{S}}(k)$ there is no such solution and only the replica symmetric solution exists ;

- if $\alpha>\alpha_{\mathrm{S}}(k)$ : if $T>T_{\mathrm{c}}$ there is no solution with $0 \leqslant m \leqslant 1$ and we should keep to the replica symmetric solution. (The restriction $0 \leqslant m \leqslant 1$ comes from the physical interpretation of $m(14)$.)

breaking; it is defined by :

if $T<T_{\mathrm{c}}$ there exists a solution at one step of replica symmetry

$$
m=\frac{T}{T_{\mathrm{c}}}, \quad q_{0}=q, \quad \hat{q}_{0}=\frac{\hat{q}}{m^{2}}, \quad q_{1}=1, \quad \hat{q}_{1}=\infty
$$

and corresponds to the following thermodynamical behaviour : the free energy is independent of $T$ and equal to the replica symmetric free energy at $T_{\mathrm{c}}$. The entropy vanishes and the energy is constant equal to the replica symmetric energy at $T_{\mathrm{c}}$. We notice that this thermodynamic behaviour is exactly identical to what is found in the simplest spin glassrandom energy model $[15,16]$, or for polymers on disordered trees [20]. However, unlike in these models, the energy levels are not independent random variables. This can be seen from the behaviour in the high temperature phase $T>T_{\mathrm{c}}$ which is not given by the annealed average. Therefore we have a somewhat new behaviour where, although the energy levels are correlated, a total freezing occurs at $T_{\mathrm{c}}$. The transition is first order in the sense that the order parameter function is not continuous, but the free energy and its first derivatives are still continuous at $T_{\mathrm{c}}$. As in the simplest spin glass, the order parameter function is a sum of two delta functions [16] : 


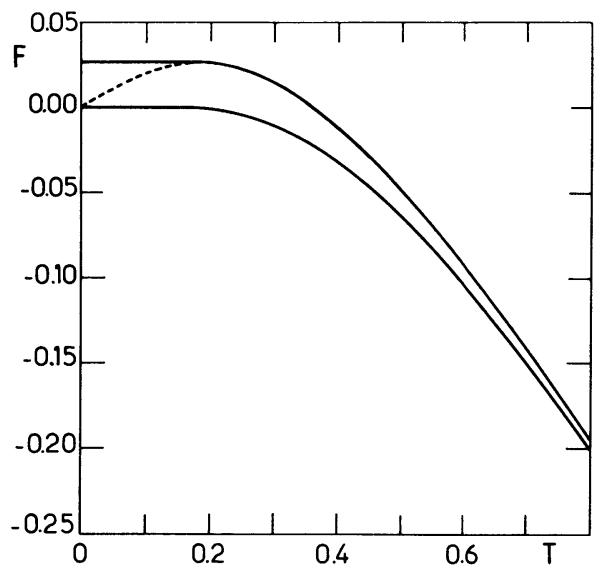

Fig. 1. - Free energy versus temperature, for $\alpha=1, \kappa=0$. The lowest curve is the annealed approximation. The upper dotted curve is the replica symmetric solution and the upper full curve is the replica symmetry breaking solution.

$$
\begin{aligned}
P(Q) & =\sum_{\alpha, \beta} \overline{P_{\alpha} P_{\beta} \delta\left(Q-\frac{1}{N} \sum_{i}\left\langle J_{i}\right\rangle_{\alpha}\left\langle J_{i}\right\rangle_{\beta}\right)} \\
& =\frac{T}{T_{\mathrm{c}}} \delta(Q-q)+\left(1-\frac{T}{T_{\mathrm{c}}}\right) \delta(Q-1)
\end{aligned}
$$

where $q$ is the replica symmetric overlap at $T_{\mathrm{c}}$.

As far as the original problem of capacity is concerned, the present solution predicts a critical capacity $\alpha=\alpha_{S}(k)$ where $\alpha_{S}(k)$ is the point where the replica symmetric entropy vanishes. The corresponding curve is plotted in figure 2.

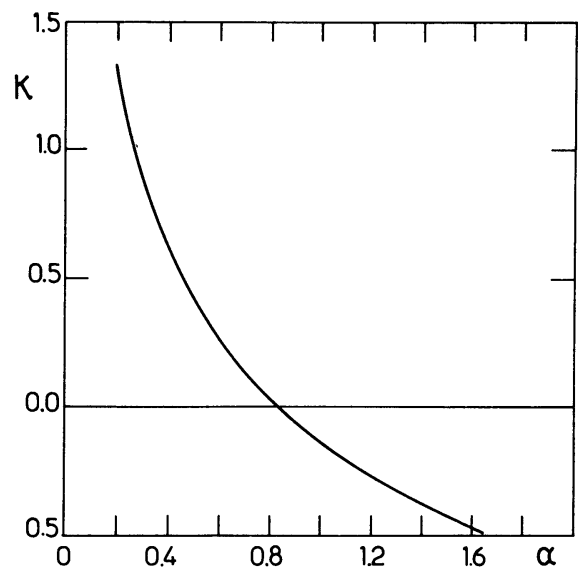

Fig. 2. - Phase diagram predicted by the one step replica symmetry breaking solution of section 6 . Shown is the critical stability $\kappa_{\mathrm{c}}$ versus the number of stored patterns per neuron $\alpha=p / N$. The system can learn in the region $\kappa \leqslant \kappa_{\mathrm{c}}(\alpha)$. The critical capacity $\alpha_{\mathrm{c}}$ is given by $\kappa_{\mathrm{c}}\left(\alpha_{\mathrm{c}}\right)=0$. 


\section{Discussion and perspectives.}

The replica symmetry breaking solution of the previous section is consistent (no negative entropy, a unique answer for the critical capacity), and in good agreement with the physical picture of section 5. It also agrees with the numerical results obtained so far in the literature [17-19]. The estimate given in [17] is $0.6<\alpha_{c}(\kappa=0)<0.9$, whereas simulations on rather small systems [19] have produced the result $\alpha_{\mathrm{c}}(\kappa=0)=0.75 \pm 0.05$, which is marginally compatible with our theoretical value. The result $\alpha_{\mathrm{c}}(\kappa)=\alpha_{\mathrm{S}}(\kappa)$ is in close agreement with the numerical findings of Krauth and Opper [18] over a wide range of values of $\alpha$; for instance it has been tested successfully for $\alpha=10$ where it gives a value of $\kappa=-1.508$ (theory) and $\kappa=-1.52 \pm 0.02$ (simulations).

An important question is whether this solution is exact or whether it is just a good approximation. As we have seen before, the stability analysis is not necessarily a good indication when there are first order transitions like here. We have rather decided to look at the problem with a second step of replica symmetry breaking à la Parisi [14]. There are then seven order parameters $q_{0}, q_{1}, q_{2}, \hat{q}_{0}, \hat{q}_{1}, \hat{q}_{2}, m_{1}, m_{2}$. In the limit $q_{2}=1, \hat{q}_{2}=\infty$, we find a result similar to (17), in the sense that the expression $G_{\mathrm{rsb}}^{(2)}$ of $1 /(N n) \log Z^{n}$ reduces to the expression $G_{\text {rsb }}^{(1)}$ we had with one step of replica symmetry breaking :

$$
G_{\mathrm{rsb}}^{(2)}\left(q_{0}, \hat{q}_{0}, q_{1}, \hat{q}_{1}, 1, \infty, m_{1}, m_{2}, \beta\right)=\frac{1}{m_{2}} G_{\mathrm{rsb}}^{(1)}\left(q_{0}, m_{2}^{2} \hat{q}_{0}, q_{1}, m_{2}^{2} \hat{q}_{1}, \frac{m_{1}}{m_{2}}, \beta m_{2}\right) .
$$

Optimizing with respect to $m_{1}$ is equivalent to optimizing with respect to $m$ in the solution of the previous section. As we said before, we have not found any solution of the equation $\partial G_{\mathrm{rsb}}^{(1)} / \partial m=0$ except if $q_{1}=1$ or $q_{1}=q_{0}$, which means that no new replica symmetry breaking occurs. This has been found numerically for $\kappa=0$ and essentially in the region where $\alpha_{\mathrm{S}} \leqslant \alpha \leqslant \alpha_{\mathrm{AT}}$ where most of our numerical work on the saddle point equations was done. Therefore we expect that at least in this region the one step r.s.b. solution of the previous section could be exact. Unfortunately this statement depends on some difficult numerical work for solving the saddle point equations at one step r.s.b. Also we have not been able so far to study the r.s.b. to all orders, as had been done in the simplest spin glass problem [16].

To summarize, we have found a one step replica symmetry breaking solution which predicts that the critical $\alpha$ is the one for which the zero temperature replica symmetric entropy vanishes. This solution might be exact, but some more analytic and numerical work to check this is still needed. Extension to biased patterns will be quite interesting.

Let us note finally that the critical line seems to be on the boarder of the replica symmetric region. Perhaps the prospects of finding a workable optimum stability algorithm are not totally bleak.

\section{Acknowledgements.}

We thank G. Toulouse for useful discussions. W. K. acknowledges financial support by Studienstiftung des deutschen Volkes. 


\section{References}

[1] Hopfield J. J., Proc. Natl. Acad. Sci. USA 79 (1982) 2554.

[2] Amit D. J., Gutfreund H. and Sompolinsky H., Phys. Rev. Lett. 55 (1985) 1530.

[3] Gardner E., Europhys. Lett. 4 (1987) 481 ; J. Phys. A 21 (1987) 257.

[4] Cover T. M., I.E.E.E. Trans. EC 14 (1965) 326.

[5] Gardner E. and Derrida B., J. Phys. A 21 (1988) 271.

[6] SOMPOLINSKy H., Heidelberg colloquium on glassy dynamics, Eds. L. van Hemmen and I. Morgenstern, Lect. Notes Phys. (Springer Verlag) 275 (1987) 485.

[7] Forrest B. M., J. Phys. A 21 (1988) 245.

[8] Krauth W., Nadal J. P. and Mézard M., J. Phys. A 21 (1988) 2995.

[9] For a review, see MÉzard M., Parisi G. and Virasoro M. A., Spin glass theory and beyond (World Scientific, Singapore) 1987.

[10] Mézard M., J. Phys. A 22 (1989) 2181.

[11] Toulouse G. and Vannimenus J., Phys. Rep. 67 (1980) 47.

[12] Krauth W. and Mézard M., J. Phys. A 20 (1987) L745.

[13] TOUlOUSE G., private communication.

[14] Parisi G., J. Phys. A 13 (1980), L115, 1101 ; see also [9].

[15] Derrida B., Phys. Rev. B 24 (1981) 2613.

[16] Gross D. J. and MÉzard M. Nucl. Phys. B 240 (1984) 431.

[17] AMAldi E. and Nicolis S., Rome.University preprint 642 (1988).

[18] Krauth W. and Opper M., J. Phys. A 22 (1989), L519.

[19] Gardner E. and Derrida B., J. Phys. A 22 (1989) 1983.

[20] DerridA B. and SpOHN H., J. Stat. Phys. 51 (1988) 817. 